\title{
Polarization anisotropy of the emission from type-II quantum dots
}

\author{
P. Klenovský, ${ }^{1,2, *}$ D. Hemzal, ${ }^{1,2}$ P. Steindl,,${ }^{1,2}$ M. Zíková, ${ }^{3}$ V. Křápek, ${ }^{4}$ and J. Humlíček ${ }^{1,2}$ \\ ${ }^{1}$ Central European Institute of Technology, Masaryk University, Kamenice 753/5, 62500 Brno, Czech Republic \\ ${ }^{2}$ Department of Condensed Matter Physics, Faculty of Science, Masaryk University, Kotlářská 2, 61137 Brno, Czech Republic \\ ${ }^{3}$ Institute of Physics CAS, Cukrovarnická 10, Praha 6, 162 00, Czech Republic \\ ${ }^{4}$ Central European Institute of Technology, Brno University of Technology, Technická 10, 61600 Brno, Czech Republic
}

(Received 16 September 2015; revised manuscript received 5 November 2015; published 15 December 2015)

\begin{abstract}
We study the polarization response of the emission from type-II GaAsSb capped InAs quantum dots. The theoretical prediction based on the calculations of the overlap integrals of the single-particle states obtained in the $\vec{k} \cdot \vec{p}$ framework is given. This is verified experimentally by polarization resolved photoluminescence measurements on samples with the type-II confinement. We show that the polarization anisotropy might be utilized to find the vertical position of the hole wave function and its orientation with respect to crystallographic axes of the sample. A proposition for usage in the information technology as a room temperature photonic gate operating at the communication wavelengths as well as a simple model to estimate the energy of fine-structure splitting for type-II GaAsSb capped InAs QDs are given.
\end{abstract}

DOI: 10.1103/PhysRevB.92.241302

PACS number(s): 78.67.Hc, 68.65.Hb, 73.21.La, 78.55.Cr

The ground state wave function of holes in type-II InAs quantum dots (QDs) with a $\mathrm{GaAs}_{1-y} \mathrm{Sb}_{y}$ capping layer (CL) resides outside of the dot volume and in general has the form of two mutually perpendicular pairs of segments [1,2]. Remarkably, the pair oriented along the [110] crystallographic direction is positioned close to the QD base, while the other, oriented along [1-10], is located above the dot [1-5]. Both the vertical position and the orientation of the hole wave function have been recently discussed in the literature [3-8]. It has been shown elsewhere [1,5] that the potential minimum for holes in this system results from a delicate interplay between quantum size effect and the piezoelectric potential and is, thus, sensitive to the thickness $d$ of CL $[3,5]$. In this work we utilize the $d$ dependence of the orientation of the hole wave function to show that the anisotropy of the polarization of the photoluminescence (PL) from this system can be used to determine the vertical position of the hole, as a gated source of radiation with defined polarization, or to estimate the fine-structure splitting energy of the exciton in type-II heterostructure.

Theory. To assess the properties of PL from type-II QDs, we have first calculated the one-particle wave functions of the InAs QDs capped by the $\mathrm{GaAs}_{1-y} \mathrm{Sb}_{y} \mathrm{CL}$ as a function of $d$ by the envelope function approach using the nextnano++ simulation suite [9], with the inclusion of the elastic strain and piezoelectricity. Second, the oscillator strengths $I_{a b}$ of the transitions between states $a$ and $b$ were calculated by a customarily built code based on the approach outlined in Refs. [10,11],

$$
I_{a b} \approx \frac{2 m_{0}}{\hbar^{2} E_{a b}}\left|\left\langle\psi_{a}|\vec{e} \cdot \hat{P}(\vec{r})| \psi_{b}\right\rangle\right|^{2},
$$

\footnotetext{
*klenovsky@physics.muni.cz

Published by the American Physical Society under the terms of the Creative Commons Attribution 3.0 License. Further distribution of this work must maintain attribution to the author(s) and the published article's title, journal citation, and DOI.
}

where $\psi_{a}$ and $\psi_{b}$ are the wave functions of the states $a$ and $b$, respectively, and $\psi=\sum_{i=1}^{8} \phi_{i} u_{i}$, where $\phi_{i}$ and $u_{i}$ are the envelope and Bloch functions for the band $i$, respectively. The other quantities are as follows: $\vec{e}$ denotes the polarization vector, $E_{a b}$ is the difference between the eigenenergies of the states $a$ and $b, \hat{P}(\vec{r})$ is the momentum operator, $m_{0}$ is the free electron mass, and $\hbar$ is the reduced Planck constant. Furthermore, because of faster spatial variations of $u$ compared to $\phi$ we make the following approximation: $\hat{P}(\vec{r}) \psi=(\hat{P}(\vec{r}) u) \phi+u(\hat{P}(\vec{r}) \phi) \approx$ $(\hat{P}(\vec{r}) u) \phi$. For more details see Refs. [10-12].

Results. We focus on the investigation of the polarization anisotropy of interband transitions, i.e., $\{a, b\}=\{c, v\}$, where $c(v)$ is the conduction (valence) band state. Including spin, the exciton transitions are formed by nearly degenerate quadruplets (bright and dark doublets) $[13,14]$ which were, however, not resolved in our experiment. Thus we calculate $I=1 / 4 \sum_{\{\uparrow, \downarrow\} \otimes\{\uparrow, \downarrow\}} I_{c v}$ only. In our experiments, both the excitation and the detected PL radiation propagate perpendicularly to the sample surface and PL is thus polarized parallel to that; the angle between the crystallographic direction [110] and the polarization vector is denoted $\alpha$. We plot our theoretical and experimental results in terms of the degree of polarization $C(\alpha)=\left[I(\alpha)-I_{\min }\right] /\left(I_{\max }+I_{\min }\right)$, where $I_{\min }$ and $I_{\max }$ are the extremal values of $I(\alpha)$. Note that for the angle $\alpha_{\max }$, such that $I\left(\alpha_{\max }\right)=I_{\max }$, the previous relation gives $C\left(\alpha_{\max }\right) \equiv$ $C_{\max }$, the maximum obtained degree of polarization.

The composition of the $\mathrm{GaAs}_{1-y} \mathrm{Sb}_{y} \mathrm{CL}$ was kept spatially constant with $\mathrm{Sb}$ content $y=0.24$ in all calculations and only $d$ was varied. Notice that we have chosen the particular value of $y$ to ensure that, except for the thinnest CLs, the confinement would be purely of type II $[1,3]$.

We have calculated the polarization properties for several dot geometries and In composition profiles, see Table I. The choice of the QD shapes was motivated by the results of Refs. [1-3,5,15-17]. Except for the structure with trumpetlike profile $[1,2,16,17]$, the values of constant In content in the QDs were chosen so as the ground state emission energy lies within the range of the communication bands, $1.3-1.55 \mu \mathrm{m}$. Based on the predicted properties of these structures, the technology of QD sample preparation was subsequently optimized to obtain 
TABLE I. Parameters of the simulated QDs along with the corresponding references. The parameters $l$ and $h$ denote the base length and height of QD, respectively. The aspect ratio is $h / l$. For the details of the trumpetlike In composition see Ref. [18].

\begin{tabular}{lcccc}
\hline \hline Shape & $l(\mathrm{~nm})$ & $h(\mathrm{~nm})$ & Aspect & In composition \\
\hline Lens [2,5,15] & 12 & 4 & 0.33 & const. In $=1$ \\
Pyramid [16] & 24 & 8 & 0.33 & const. In $=0.6$ \\
Pyramid [1,17] & 24 & 8 & 0.33 & trumpetlike \\
Trunc. cone & 24 & 6.5 & 0.27 & const. In $=0.8$ \\
Trunc. pyr. [3] & 24 & 6.5 & 0.27 & const. In $=0.8$ \\
\hline \hline
\end{tabular}

QDs with desired properties, i.e., the long emission wavelength and type-II band alignment. Typical results of our calculations are shown in Fig. 1 for the lens-shaped QD with the base diameter and height of 12 and $4 \mathrm{~nm}$, respectively, and two CL thicknesses of $d=3 \mathrm{~nm}$ and $d=12 \mathrm{~nm}$. Notably, the emitted light is preferentially polarized along the [110] ([1-10]) crystallographic direction for thin (thick) CL. The dominant contribution to $I(\alpha)$ in QDs comes from the areas with the largest overlap between electrons and holes. The electron is firmly bound in the body of type-II QDs for all Sb contents and CL thicknesses [1,3]. Thus, also due to the larger effective mass of holes the overlap and the resulting polarization anisotropy of $I(\alpha)$ is dictated by those parts of the hole wave function which are located closest to the dot, see Fig. 1(a).
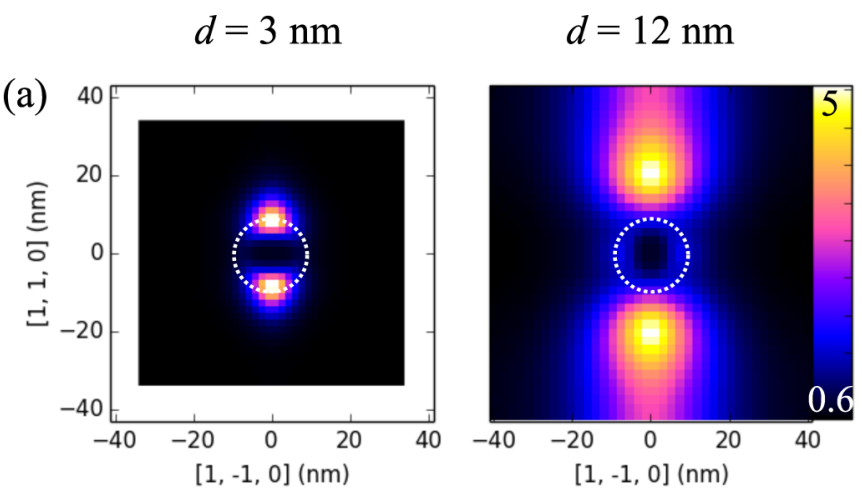

(b)
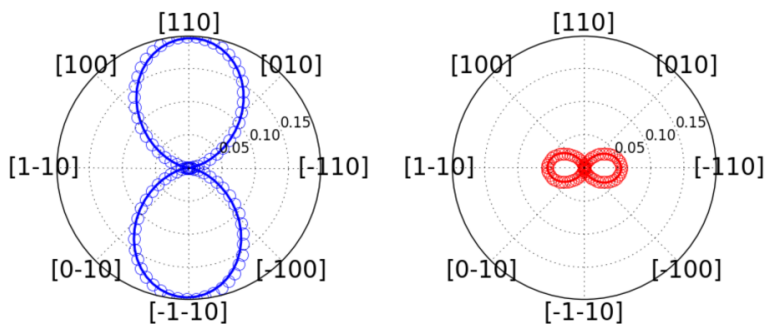

FIG. 1. (Color online) (a) Horizontal cross section of the hole probability density for the lens-shaped QD with a base diameter and height of 12 and $4 \mathrm{~nm}$, respectively, and for CL thicknesses of $d=$ $3 \mathrm{~nm}$ and $d=12 \mathrm{~nm}$. The color scale in (a) is in units of $10^{-5} \mathrm{~m}^{-3}$. The cuts cross the position of the maximum of the probability density at $1.8 \mathrm{~nm}(6.0 \mathrm{~nm})$ above the dot base for CL thickness of $3 \mathrm{~nm}$ (12 nm). (b) The calculated $C(\alpha)$ for the ground state electron-hole transition; full curve is a guide to the eye. Dotted white circles in (a) mark the edges of the dot. The electron wave function occupies the dot volume and is slightly elongated in the [1-10] direction.

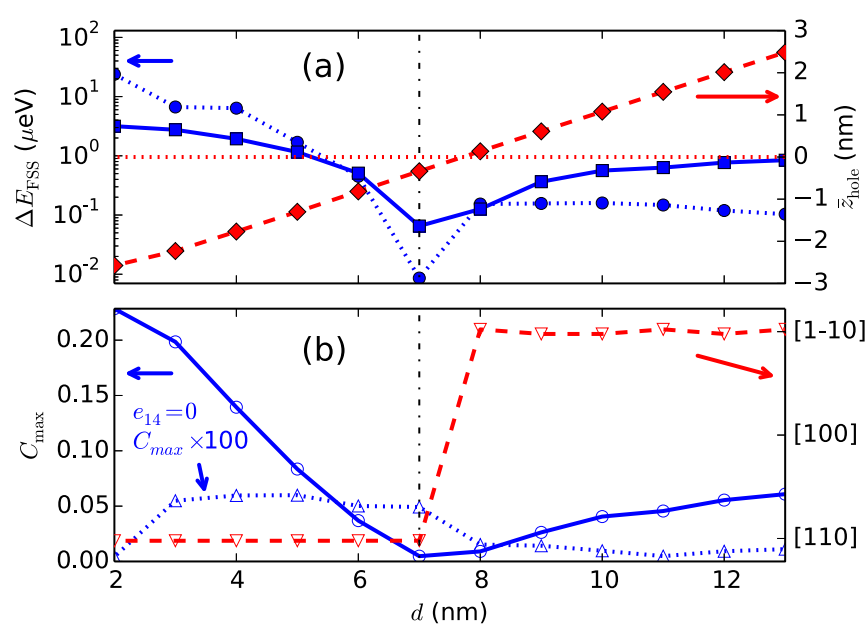

FIG. 2. (Color online) (a) Calculated $d$ dependencies of $\Delta E_{\mathrm{FSS}}$ estimated from $C_{\max }$ (full squares) that were calculated by the model of Ref. [5] (full circles), and the relative vertical position of the hole $\bar{z}_{\text {hole }}$ (full diamonds). (b) $d$ dependencies of $C_{\max }$ (open circles) with piezo switched off and multiplied by a factor of 100 (upward open triangles) and $\alpha_{\max }$ (downward open triangles). The latter is shown in the crystallographic axes of the QD material. Calculations were done for lens-shaped QD, see Table I. The vertical dot-dash line indicates $d_{c}$. The remaining curves are guides to the eye.

We show in Fig. 2(a) that with $d$ increasing, the vertical position of the hole wave function is first shifted upwards and the average of the vertical hole coordinate measured from the top of the $\mathrm{QD}$, denoted $\bar{z}_{\text {hole }}$, increases. At the same time $C_{\max }$ is reduced [Fig. 2(b)] until, for certain critical CL thickness $d_{c}$, the emission becomes isotropic $\left(C_{\max }=0\right)$ and the average hole vertical position coincides with the top of the QD $\left(\bar{z}_{\text {hole }}=0\right)$. During this period also $\alpha_{\max }=0^{\circ}$ holds and the hole segments are oriented along the [110] direction. However, further increase of $d$ results in an increase of $\bar{z}_{\text {hole }}$ and $C_{\max }$. Also a flip of the orientation $\alpha_{\max }$ to [1-10] direction for $d_{c}$ is observed and this is attained for even larger values of $d$. While $\alpha_{\max }$ is identical with the orientation of the dominant pair of segments of the hole wave function, i.e., those with the largest overlap with the electrons, $C_{\max }$ corresponds to the ratio of overlaps of both pairs with them, see also Fig. 1(a). The correspondence of $C_{\max }$ is, however, not exact because it is influenced also by a slight elongation of the electron wave function, occurring along [1-10].

The overlap of electrons and holes is rather small in type-II QDs $[1,2,19]$. However, particularly for GaAsSb capped InAs QDs we can take advantage of that to estimate the energy of the fine-structure splitting $\left(\Delta E_{\mathrm{FSS}}\right)$, i.e., the energy separation of the bright excitonic doublet, from $C_{\max }$. Motivated by the results of Ref. [5] we estimate $\Delta E_{\mathrm{FSS}}$ as

$$
\Delta E_{\mathrm{FSS}} \sim \frac{e^{2}}{2 \pi \varepsilon \bar{l}_{\mathrm{QD}}^{3}} C_{\mathrm{max}},
$$

where $e$ is the elementary charge, $\varepsilon$ is the permittivity, and $\bar{l}_{\mathrm{QD}}$ is the mean diameter of the QD base. The length $\bar{l}_{\mathrm{QD}}$ replaces $\left|\vec{r}_{e}-\vec{r}_{h}\right|$, where $\vec{r}_{e}\left(\vec{r}_{h}\right)$ is the position of the electron (hole). In constructing $\bar{l}_{\mathrm{QD}}$ we have noted a typical situation for type-II GaAsSb capped InAs QDs where the maximum of probability 
densities for the electron and hole is positioned at similar distances from the edge of the dot. To make the estimation even easier we considered the QD to have the shape of a hemisphere with an electron located in the center of its circular base. Hence $\bar{l}_{\mathrm{QD}}$ is in turn equal to the diameter of the hemisphere's base. Equation (2) provides a simple, rough estimate of $\Delta E_{\mathrm{FSS}}$ from the measured values of $C_{\max }$ and the structural parameters of QDs. The latter can be obtained by standard structural characterization tools. For further information on Eq. (2) see Ref. [18]. The comparison of Eq. (2) to more elaborate calculations of $\Delta E_{\mathrm{FSS}}$, see Ref. [5] for details, is shown in Fig. 2(a). The error is as large as an order of magnitude. Evidently, Eq. (2) gives most precise estimates of $\Delta E_{\mathrm{FSS}}$ for small values of $C_{\max }$. The value of $\alpha_{\max }$ is identical with that for the lower energy exciton of the corresponding exciton doublet.

We have calculated $\alpha_{\max }$ and $C_{\max }$ also for other QD shapes and In composition from Table I and confirmed the existence of $d_{c}$ associated with the vertical position of the (ground state) hole wave function reaching the QD apex also for pyramidal QDs. Only in truncated shapes are the hole segments oriented along [110] for all $d$ values in agreement with the results of Ref. [3]. We note that the error of the estimate of $\Delta E_{\mathrm{FSS}}$ was the same for all studied QDs listed in Table I.

It is the correspondence between the orientation of its segments and the vertical position of the hole wave function with respect to the QD volume that enables us to determine its position for untruncated QD shapes in real experiments simply by measuring the direction of the in-plane PL polarization anisotropy.

The behavior just described is closely connected to the piezoelectricity induced by the shear stress as can be seen from the dependence of $C_{\max }$ on $d$ being more than hundred times weaker when the piezoelectric term $e_{14}$ is set to zero in the calculations, see the dash-dotted curve in Fig. 2(b). We note that the effect of the shear elements of the Bir-Pikus strain Hamiltonian [20] via the deformation potentials is negligible as well.

The quantities $C_{\max }$ and $\alpha_{\max }$ have an intimate relation to the mixing of heavy (HH) and light ( $\mathrm{LH})$ hole states as that is responsible for the shape and orientation of the envelope wave function of the hole [21,22]. For our dots the phase of HH-LH mixing determines $\alpha_{\max }$, and its amplitude $C_{\max }$. Both parameters can be determined using the results of Refs. $[23,24]$.

To test our theoretical predictions, three samples of InAs QDs with GaAsSb CL, denoted A, B, and C, were fabricated. In view of the possible future applications, the low pressure metal-organic vapor phase epitaxy (MOVPE) growth was chosen [25]. We have prepared the samples so that the amount of $\mathrm{Sb}$ in $\mathrm{CL}$ would correspond to type-II band alignment, which was confirmed by the blueshift of PL with increased pumping, see Ref. [18]. The InAs QD layer was grown under different V/III ratios for each sample. More information about sample preparation can be found in the Supplemental Material [18]. Different conditions of sample preparation along with the nonuniform growth on the nonrotating susceptor provided us with the required variety of QDs with different sizes, shapes, and CLs with variable Sb contents and thicknesses.

The measurements of the polarization anisotropy of PL were performed using the NT-MDT Ntegra-spectra spectrometer. The samples were positioned in the cryostat and cooled
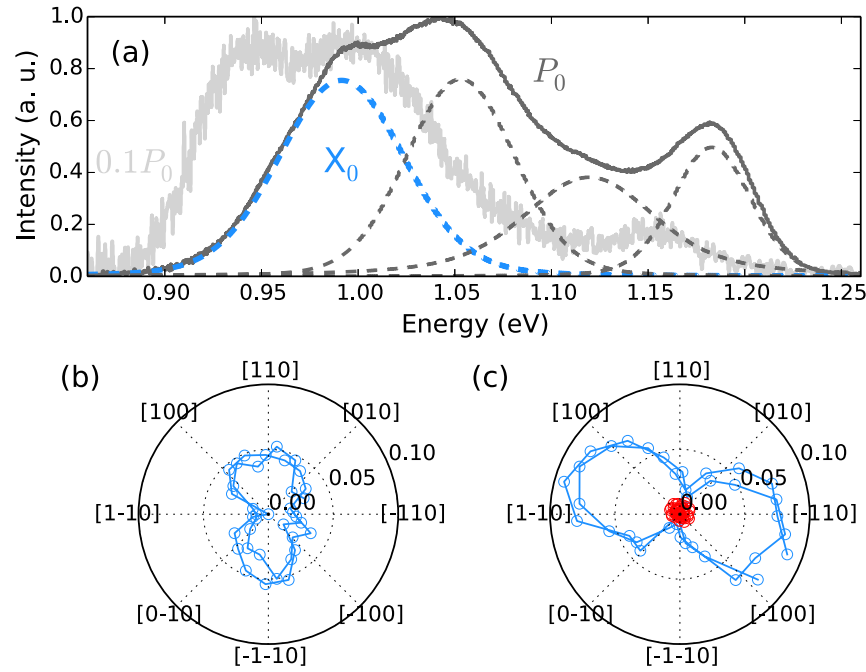

FIG. 3. (Color online) (a) PL spectra measured at LN2 temperature of sample $\mathrm{C}$ for pumping powers of $P_{0}=14 \mathrm{kWcm}^{-2}$ (dark gray curve) and $1.4 \mathrm{~kW} \mathrm{~cm}^{-2}$ (light gray curve). Notice the blueshift of $50 \mathrm{meV}$ between the spectra. For the larger excitation power we also show the individual fitted Gauss-Lorentz bands by broken curves and that with the lowest energy (blue broken curve, denoted by $\mathrm{X}_{0}$ ) for which we analyzed the polarization anisotropy. (b) and (c) Polarization dependence of the intensity of the lowest-energy PL band for samples $\mathrm{A}$ and $\mathrm{C}$, respectively (blue circles). The polarization angles are represented by the corresponding crystallographic axes. Note that the lowest transitions are polarized perpendicularly from one sample to the other. The red circles represent the residual polarization anisotropy of the setup which was 0.005 . In (b) and (c) the lines are guides to the eye.

to liquid nitrogen temperature (LN2) (for room temperature PL results see Ref. [18]) and were pumped by the solid-state laser with the wavelength of $785 \mathrm{~nm}$ and maximum power on the sample surface of $30 \mathrm{~mW}$, which was varied by a tunable neutral density (ND) filter. In every experiment we have collected PL light from a large number of QDs ( 2000) in order to damp the deviations from the mean properties of the ensemble of dots. The polarization of PL was analyzed by a rotating half-wave plate followed by a fixed linear polarizer. Finally, the PL signal was dispersed by a 150 grooves $/ \mathrm{mm}$ ruled grating and detected by the InGaAs line-CCD camera, cooled to minus $90^{\circ} \mathrm{C}$.

The experimental procedure was as follows. First, the type of confinement was determined by measuring the pumping dependency of the PL spectra. Either a blueshift of the whole spectrum was observed, indicating type-II confinement [6,19], or the spectrum only increased in magnitude without any visible spectral shift, and we considered it as type I. For a typical example of the results obtained on sample $\mathrm{C}$ see Fig. 3(a). Consequently, the PL spectra for 37 angular positions of $\lambda / 2$ plate at fixed laser pumping power were acquired and fitted by a sum of Gauss-Lorentz (GL) profiles [26], see an example of the decomposition in Fig. 3(a). As our calculations described above are most accurate for the ground state transition, we consider here the intensity of the GL band with the lowest energy only. Examples are shown in Figs. 3(b) and 3(c) for type-II samples A and C. Note that the dominant polarization direction for the lowest-energy transition is clearly along [110] 


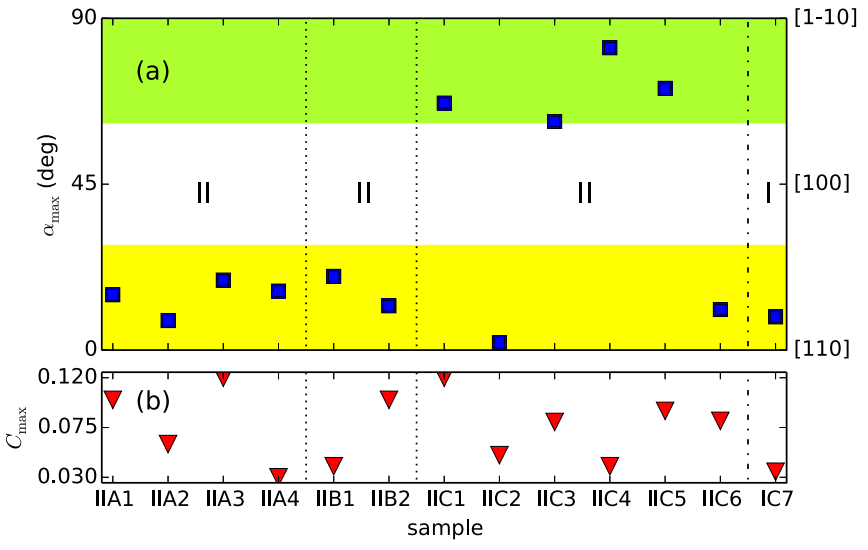

FIG. 4. (Color online) (a) Measured $\alpha_{\max }$ reduced to the first quadrant (blue squares), and (b) $C_{\max }$ (red triangles) for three samples (A-C) and different positions on them, labeled by $x y z$, where $x$ refers to the type of confinement, $y$ to the sample, and $z$ to the position on it. The dashed vertical lines mark different samples and the dash-dotted one a different type of confinement which is also denoted by I or II in (a). The green/yellow stripes in (a) are the typical errors of $\alpha_{\max }$ of $30^{\circ}$. The right vertical axis in (a) represents the crystallographic axes of the sample.

in (b), and close to [1-10] in (c). We have observed the polarization anisotropy also for the other GL bands and its degree was found to be increasing with the emission energy, consistent with the admixture of the excited states. We stress that due to expected small values of $C_{\max }$ we paid a particular attention to (i) the reduction of the residual polarization of the whole setup, see red circles in Fig. 3(c), and (ii) proper fitting of the spectra.

The resulting $\alpha_{\max }$ for all samples and different positions on them are summarized in Fig. 4(a). For type II we have observed $\alpha_{\max }$ corresponding to both [110] and [1-10] crystallographic directions, the former being more frequent. This in turn means that holes were located preferentially close to the base of the QD. Furthermore, for type I $\alpha_{\max }$ corresponded to [110] direction, in agreement with the results on InAs/GaAs QDs [27]. We note, however, that the spread of the obtained values of $\alpha_{\max }$ might be as large as $30^{\circ}$. We assume that this is an effect of the QD and CL irregularities which were still not averaged out and also due to remaining imperfections of the measurement procedure.

The measured values of $C_{\max }$ are given in Fig. 4(b) and are varying from 0.03 to 0.12 . Clearly type-II GaAsSb capped InAs QDs might possess a significant polarization anisotropy.

Finally, the nature of the studied effect opens a number of possibilities to tune $\alpha_{\max }$ and $C_{\max }$ by external fields, e.g., electric or strain $[1,21,28]$. In the information technology this could enable the two perpendicular polarizations of the emitted photons to serve as a low-drain photonic realization of "zeros" and "ones," with the bonus of operation at communication wavelengths. In addition, this behavior can be reached for large ensembles of QDs, which was demonstrated here by measurements on the MOVPE-prepared samples, and even at room temperature.

Conclusions. We have theoretically predicted and also experimentally observed two perpendicular PL polarizations of type-II GaAsSb capped InAs QDs and explained it as an almost exclusive effect of the piezoelectricity. The measurement of polarization anisotropy enables the determination of the vertical position of the holes and their orientation in the studied system. Furthermore, a simple relation to estimate the energy of FSS in type-II QD structures and the utilization of the polarization anisotropy as a room-temperature gate based on photons with energy in the communication bands and defined polarization state were proposed.

Acknowledgments. The authors thank Alice Hospodková for her help with the sample preparation and fruitful discussions. The work was supported by the project No. TH01010419 of the Technological agency of the Czech Republic, the European Regional Development Fund, project No. CZ.1.05/1.1.00/02.0068, and the European Social Fund (Belgium), Grant No. CZ.1.07/2.3.00/30.0005.
[1] P. Klenovský, V. Křápek, D. Munzar, and J. Humlíček, Appl. Phys. Lett. 97, 203107 (2010).

[2] P. Klenovský, V. Křápek, D. Munzar, and J. Humlíček, J. Phys. Conf. Ser. 245, 012086 (2010).

[3] W.-T. Hsu, Y.-A. Liao, F.-C. Hsu, P.-C. Chiu, J.-I. Chyi, and W.-H. Chang, Appl. Phys. Lett. 99, 073108 (2011).

[4] J. M. Ulloa, R. Gargallo-Caballero, M. Bozkurt, M. del Moral, A. Guzman, P. M. Koenraad, and A. Hierro, Phys. Rev. B 81, 165305 (2010).

[5] V. Křápek, P. Klenovský, and T. Šikola, Phys. Rev. B 92, 195430 (2015).

[6] C. Y. Jin, H. Y. Liu, S. Y. Zhang, Q. Jiang, S. L. Liew, M. Hopkinson, T. J. Badcock, E. Nabavi, and D. J. Mowbray, Appl. Phys. Lett. 91, 021102 (2007).

[7] H. Liu, M. Steer, T. Badcock, D. Mowbray, M. Skolnick, P. Navaretti, K. Groom, M. Hopkinson, and R. Hogg, Appl. Phys. Lett. 86, 143108 (2005).

[8] J. M. Ulloa, J. M. Llorens, B. Alen, D. F. Reyes, D. L. Sales, D. Gonzalez, and A. Hierro, Appl. Phys. Lett. 101, 253112 (2012).
[9] S. Birner, T. Zibold, T. Andlauer, T. Kubis, M. Sabathil, A. Trellakis, and P. Vogl, IEEE Trans. Electron Devices 54, 2137 (2007).

[10] O. Stier, Ph.D. thesis, Technische Universität Berlin, 2000.

[11] O. Stier, M. Grundmann, and D. Bimberg, Phys. Rev. B 59, 5688 (1999).

[12] T. Takagahara, Phys. Rev. B 62, 16840 (2000).

[13] M. Bayer, G. Ortner, O. Stern, A. Kuther, A. A. Gorbunov, A. Forchel, P. Hawrylak, S. Fafard, K. Hinzer, T. L. Reinecke, S. N. Walck, J. P. Reithmaier, F. Klopf, and F. Schafer, Phys. Rev. B 65, 195315 (2002).

[14] M. Bayer, A. Kuther, A. Forchel, A. Gorbunov, V. B. Timofeev, F. Schafer, J. P. Reithmaier, T. L. Reinecke, and S. N. Walck, Phys. Rev. Lett. 82, 1748 (1999).

[15] A. Hospodková, M. Zíková, J. Pangrác, J. Oswald, J. Kubištová, K. Kuldová, P. Hazdra, and E. Hulicius, J. Phys. D 46, 095103 (2013).

[16] J. M. Ulloa, I. W. D. Drouzas, P. M. Koenraad, D. J. Mowbray, M. J. Steer, H. Y. Liu, and M. Hopkinson, Appl. Phys. Lett. 90, 213105 (2007). 
[17] P. Offermans, P. M. Koenraad, J. H. Wolter, K. Pierz, M. Roy, and P. A. Maksym, Phys. Rev. B 72, 165332 (2005).

[18] See Supplemental Material at http://link.aps.org/supplemental/ 10.1103/PhysRevB.92.241302 for details on the calculations and sample preparation.

[19] P. Klenovský, M. Brehm, V. Křápek, E. Lausecker, D. Munzar, F. Hackl, H. Steiner, T. Fromherz, G. Bauer, and J. Humlíček, Phys. Rev. B 86, 115305 (2012).

[20] G. L. Bir and G. E. Pikus, Symmetry and Strain-Induced Effects in Semiconductors (Wiley, New York, 1974).

[21] J. D. Plumhof, V. Křápek, F. Ding, K. D. Jons, R. Hafenbrak, P. Klenovský, A. Herklotz, K. Dorr, P. Michler, A. Rastelli, and O. G. Schmidt, Phys. Rev. B 83, 121302 (2011).

[22] A. Musial, P. Kaczmarkiewicz, G. Sek, P. Podemski, P. Machnikowski, J. Misiewicz, S. Hein, S. Hofling, and A. Forchel, Phys. Rev. B 85, 035314 (2012).
[23] C. Tonin, R. Hostein, V. Voliotis, R. Grousson, A. Lemaitre, and A. Martinez, Phys. Rev. B 85, 155303 (2012).

[24] Y. Leger, L. Besombes, L. Maingault, and H. Mariette, Phys. Rev. B 76, 045331 (2007).

[25] A. Hospodková, J. Vyskočil, J. Pangrác, J. Oswald, E. Hulicius, and K. Kuldová, Surf. Sci. 604, 318 (2010).

[26] J. Humlíček, E. Schmidt, L. Bočánek, R. Švehla, and K. Ploog, Phys. Rev. B 48, 5241 (1993).

[27] J. Humlíček, D. Munzar, K. Navrátil, M. Lorenc, J. Oswald, J. Pangrác, and E. Hulicius, Phys. E 13, 229 (2002).

[28] R. Trotta, P. Atkinson, J. D. Plumhof, E. Zallo, R. O. Rezaev, S. Kumar, S. Baunack, J. R. Schroeter, A. Rastelli, and O. G. Schmidt, Adv. Mater. 24, 2668 (2012). 